\title{
Integración sociopolítica y bienestar subjetivo en México ${ }^{1}$
}

René Millán

\section{I ntroducción}

Pese a ser un campo relativamente nuevo, la investigación sobre bienestar subjetivo (BS) se ha ido consolidando rápidamente a nivel mundial y, pese a ciertas resistencias, también en América Latina ${ }^{2}$. En el marco de la investigación, un buen número de estudios han asociado al BS con distintas variables que van desde la salud (Florenzano y Dussaillant, 2011), la familia (Gardner y Osward, 2006) hasta la equidad social (Alesina, Di Tella y MacCulloch, 2004). Hay también análisis que han identificado la importancia de las relaciones humanas y sociales (Bartolini, 2012; Helliwell y Barrington-Leigh, 2011). En este sentido, el interés de este artículo es mostrar que las formas de integración sociopolítica, así como las pautas de cohesión social a ellas vinculadas, también influyen sustantivamente en dicho bienestar. Esa influencia explica en parte el diferente peso o significación que variables vinculadas con pautas de integración social y política adquieren en distintos contextos. En otros términos, la tesis de este artículo es que si bien existen ciertas variables que se asocian al BS independientemente de los contextos sociales (como la salud), hay otras cuya importancia está fuertemente determinada por esos contextos y su comportamiento está asociado a las formas de integración sociopolítica.

Este estudio se basa en una encuesta que se realizó en $2006^{3}$ en dos ciudades mexicanas con características distintas: Chilpancingo (CHP) y Monterey (MTY). Para

\footnotetext{
${ }^{1}$ Este artículo fue posible gracias al apoyo de Conacyt.

2 Como se sabe, el BS se mide con dos variables cercanas pero que mantienen cierta diferencia conceptual: la felicidad y la satisfacción con la vida. En general se asume que la primera implica sobre todo aspectos de tipo afectivo o de equilibrio emocional y la segunda a dimensiones cognitivas y evaluativas. Sin embargo, en términos empíricos tienden a comportarse de manera muy semejante. En la medición de las variables es el individuo quien auto-reporta sus niveles de felicidad o satisfacción normalmente en una escala de uno a diez, aunque también se mide en escalas de 1 a 5 o 1 a 7 . Para un buen análisis de lo publicado en América Latina sobre BS, su conceptualización y las formas de medirlo y variables asociadas véase: Rojas y Martínez (2012). Para los acuerdos sobre medición y conceptualización véase OCDE (2013).

${ }^{3}$ Para cada uno de las ciudades, considerados como dominio de estudio independientes. El tamaño de muestra calculado para cada uno de los dominios de estudio fue de 1,072 casos, con un $95 \%$ de confianza, error de estimación no mayor de cuatro puntos porcentuales y tasa de no respuesta de $5 \%$.
} 
2004-2005, MTY registraba un Índice de Desarrollo Humano considerablemente alto para el país $(0,853)$, con un PIB per cápita de 14.769 dólares y un Índice de Marginalidad (IM) muy bajo (1,676). CHP, en cambio, alanzaba IDH medio alto $(0,789)$, con 5.419 dólares de PIB per cápita y un IM bajo $(1,310)^{4}$. Además, en MTY se encuentra al norte del país y ha sido un centro de desarrollo industrial y de servicios, con valores más empresariales, con menos intervención estatal en la configuración de la cultura política local y en la economía. CHP contaba y cuenta con una estructura industrial menor y muy ligada a las actividades del agro. En considerable grado, la cultura política o cívica reproduce todavía hoy pautas clientelares entre gobierno y sociedad. CHP cuenta con asociaciones sociales o sindicatos fuertes que tienen como principal interlocutor a las autoridades locales o federales.

Para hacer plausible la tesis que he postulado, muestro, primero, los resultados de la investigación y luego realizo una discusión sobre esos resultados, variable por variable, para mostrar como su comportamiento puede referirse conceptualmente a la integración sociopolítica.

\section{Resultados de la investigación}

Para el análisis del BS utilizamos la categoría de felicidad medida en una escala de cinco valores que, una vez recodificados, quedaron en dos categorías (feliz/no feliz) ${ }^{5}$. En ambas ciudades el porcentaje de personas que se auto-reportaron como felices fue considerable: $74,2 \%$, en CHP; $84 \%$, en MTY. Para cada una de las ciudades se realizó un análisis multivariado. Originalmente exploramos un conjunto grande de variables que corresponden, conceptualmente, a cuatro dimensiones de la integración sociopolítica:

\footnotetext{
${ }^{4}$ Como contraste, el país tenía un IDH de 0,807; un PIB per cápita de 6.770 dólares. No hay dato sobre el IM nacional, pues se reporta por municipios o entidad federativa, véase sitio Consejo Nacional de Población (Conapo): <www.conapo.gob. $\mathrm{mx}>$.

5e preguntó: "En una escala del 1 al 5, como en la escuela, que tan feliz es Usted?". Se consideraron felices los valores 4 y 5 , y como no felices los restantes.
} 
Variables de la integración sociopolítica

\begin{tabular}{|c|c|c|c|}
\hline Cohesión social & Capital social & Ciudadanía y gobierno & Calidad de vida \\
\hline $\begin{array}{l}\text { 1. Conflicto con } \\
\text { familiares } \\
\text { 2. Conflicto con amigos } \\
\text { 3. Conflicto con vecinos } \\
\text { 4. Fragmentación social } \\
\text { 5. Posibilidad de } \\
\text { nuevas relaciones }\end{array}$ & $\begin{array}{l}\text { 6. Confianza } \\
\text { 7. Reciprocidad } \\
\text { 8. Participación en } \\
\text { asociaciones } \\
\text { voluntarias } \\
\text { 9. Cooperación } \\
\text { 10.Bienes relacionales } \\
\text { (confianza en la } \\
\text { familia y sociabilidad } \\
\text { con amigos) }\end{array}$ & $\begin{array}{l}\text { 12. Participación } \\
\text { electoral } \\
\text { 13. Interés en los } \\
\text { asuntos públicos } \\
\text { 14. Respeto a los } \\
\text { derechos }\end{array}$ & $\begin{array}{l}\text { 15. Habitabilidad } \\
\text { 16. Ingreso }\end{array}$ \\
\hline
\end{tabular}

Fuente: Encuesta propia.

Como se observará enseguida, el modelo fue ajustado porque ciertas variables que en teoría deberían estar asociadas al BS no se comportaron conforme a esa expectativa. Por su implicación teórica, pero sobre todo porque su falta de asociación con el BS refuerza la importancia del análisis contextual dada su referencia en la literatura especializada, algunas de ellas son incorporadas en la reflexión que sigue pero no en los cuadros de regresión (Cuadros 1 y 2 ).

En términos generales, el resultado del análisis comparado, como sugeríamos antes, sugiere que el contexto sociopolítico es un factor importante en el comportamiento heterogéneo de las variables respecto de la BS. La heterogeneidad puede tener razones individuales y sociales. Para Diener y Oishi (2000), por ejemplo, diferencias culturales y sociales explican la disparidad internacional en el comportamiento del BS. Para Rojas y Vitterso (2010, p. 1) "conceptions of happiness differ not only across people, but also across cultures, since the conception one holds is not independent of the specific personal and social context one lives in and grew up". Para Helliwell, Layard y Sachs (2013, p. 2) estudiar tanto el nivel individual como el social ayudaría a explicar las diferencias entre sociedades distintas. La heterogeneidad que reporta este artículo surge de las características singulares de cada ciudad y ofrece una oportunidad para mostrar que muchas de las variables que normalmente aparecen asociadas al BS en los análisis internacionales no se comportan de ese modo. Esto se mostrará en el análisis de las cuatro dimensiones de la integración sociopolítica que presentamos a continuación. 
Cuadro 1

Chilpancingo. Resultados de la aplicación de un modelo de regresión logística binario para estimar la probabilidad de ser feliz

\begin{tabular}{|c|c|c|c|c|c|}
\hline Variables & B & Sig. & S.E. & Wald & Exp (B) \\
\hline Edad del entrevistado & $-0,092$ & $* *$ & 0,032 & 8,215 & 0,912 \\
\hline Edad al cuadrado & 0,001 & $* *$ & 0,000 & 3,987 & 1,001 \\
\hline Conflicto con vecinos & $-0,697$ & $* *$ & 0,228 & 9,321 & 0,498 \\
\hline Í ndice de fragmentación social & $-0,666$ & $* *$ & 0,247 & 7,301 & 0,514 \\
\hline Establecer nuevas relaciones & 0,369 & $*$ & 0,209 & 3,107 & 1,446 \\
\hline Confianza en la familia & 0,863 & $* * *$ & 0,216 & 16,004 & 2,369 \\
\hline Personas se tratan como iguales & 0,427 & $* *$ & 0,201 & 4,54 & 1,533 \\
\hline Cooperación en proyecto colectivo & 0,422 & $* *$ & 0,236 & 3,206 & 1,526 \\
\hline $\begin{array}{l}\text { I nterés en asuntos políticos del } \\
\text { país }\end{array}$ & & $* *$ & & 15,227 & \\
\hline Interesado & $-0,201$ & & 0,476 & 0,178 & 0,818 \\
\hline Más o menos interesado & $-1,222$ & $* *$ & 0,529 & 5,332 & 0,295 \\
\hline Poco interesado & $-1,037$ & $* *$ & 0,438 & 5,596 & 0,354 \\
\hline Nada interesado & $-1,057$ & $* *$ & 0,455 & 5,407 & 0,347 \\
\hline $\begin{array}{l}\text { Gobierno considera opinión de la } \\
\text { gente }\end{array}$ & & $* * *$ & & 16,705 & \\
\hline Indiferente & $-1,475$ & $* * *$ & 0,377 & 15,321 & 0,229 \\
\hline Poco o nada & $-0,669$ & $* *$ & 0,234 & 8,136 & 0,512 \\
\hline Satisfacción lugar donde vive & & $* *$ & & 8,011 & \\
\hline Regular & $-0,478$ & $* *$ & 0,195 & 6,023 & 0,620 \\
\hline Malo & $-1,010$ & $* *$ & 0,592 & 2,908 & 0,364 \\
\hline I ngresos del hogar & & $* * *$ & & 30,15 & \\
\hline Más de dos y menos de cuatro s.m. & 0,607 & $* *$ & 0,203 & 8,995 & 1,836 \\
\hline De cuatro a seis s.m. & 1,313 & $* * *$ & 0,342 & 14,703 & 3,715 \\
\hline Más de seis s.m. & 1,423 & $* * *$ & 0,309 & 21,178 & 4,148 \\
\hline Constante & 3,234 & $* * *$ & 0,835 & 14,982 & \\
\hline
\end{tabular}

Fuente: Elaboración propia.

Significancia: * $\mathrm{p}<0.10$; ** $\mathrm{p}<0.05$; *** $\mathrm{p}<0.000$

Chi cuadrada del modelo: (189.15 $19 \mathrm{gl}$ ) Sig. .000

-2loglikelihood: 781,56 . 


\section{Cuadro 2}

Monterrey. Resultados de la aplicación de un modelo de regresión logística binario para estimar la probabilidad de ser feliz

\begin{tabular}{|l|c|c|c|c|c|}
\hline \multicolumn{1}{|c|}{ Variables } & B & Sig. & S.E. & Wald & Exp (B) \\
\hline Edad del entrevistado & & & & & \\
\hline & $-0,372$ & $*$ & 0,201 & 3,425 & 0,689 \\
\hline Confianza en la familia & & & & & \\
\hline Personas se tratan como iguales & 0,513 & $*$ & 0,268 & 3,655 & 1,670 \\
\hline & & & & & \\
\hline Índice de contactos con amigos & 0,423 & $*$ & 0,241 & 3,085 & 1,527 \\
\hline & & & & & \\
\hline Respetan sus derechos & 0,603 & $* *$ & 0,237 & 6,495 & 1,828 \\
\hline No & & & & & \\
\hline Algunos sí otros no & & $* *$ & & 9,244 & \\
\hline & $-0,407$ & $*$ & 0,232 & 3,073 & 0,666 \\
\hline Satisfacción lugar donde vive & 0,351 & & 0,280 & 1,565 & 1,420 \\
\hline Regular & & & & & \\
\hline Malo & & $* * *$ & & 29,649 & \\
\hline & $-1,040$ & $* * *$ & 0,209 & 24,634 & 0,354 \\
\hline Ingresos del logar & $-1,461$ & $* * *$ & 0,417 & 12,298 & 0,232 \\
\hline Más de dos y menos de cuatro s.m. & & & & & \\
\hline De cuatro a seis s.m. & & $* *$ & & 13,326 & \\
\hline Más de seis s.m. & 0,114 & & 0,260 & 0,192 & 1,121 \\
\hline & 0,839 & $* *$ & 0,384 & 4,774 & 2,314 \\
\hline Constante & 0,858 & $* *$ & 0,306 & 7,866 & 2,359 \\
\hline Fuente: & & & & & \\
\hline
\end{tabular}

Fuente: Elaboración propia.

Significancia: $* \mathrm{p}<0.10 ; * * \mathrm{p}<0.05 ; * * * \mathrm{p}<0.000$

Chi cuadrada del modelo: 100.245 (11gl); Sig. .000

-2loglikelihood: 686,958 


\section{Cohesión social}

El carácter polisémico del concepto es reconocido (Ottone, 2007). Pese a esa dificultad, creemos que una sociedad cohesionada tiene, respecto de aquella que no lo está, un recurso adicional para la solución de sus problemas. De ahí que nos preocupe, aunque no siempre designamos lo mismo con el concepto. En los últimos años tanto el European Committee for Social Cohesion (2004) como la Cepal (2007) han utilizado como "meta concepto" que opera como un equivalente de inclusión social en distintas esferas de la vida asociada (trabajo, educación, servicios). Bajo esa amplitud, se utiliza como un referente para evaluar el desempeño general de la sociedad. Ese uso ha debilitado el sentido original del término. Para efectos del BS es más útil volver a noción original y "restringida" del concepto.

La utilidad analítica de la cohesión social es atribuida a Durkheim (1960) en su célebre distinción entre tipos de solidaridad. En las sociedades simples, "mecánicas", la cohesión se logra gracias a una fuerte y robusta "conciencia colectiva" que diluye las tensiones y conflictos al restringir el rango de experiencias de los individuos dada la escasa división del trabajo. En las complejas y "orgánicas", debe asumirse esa tensión, al menos como principio, debido al incremento notable de la especialización de funciones y ámbitos de experiencia. La tensión se resuelve por el reforzamiento de aspectos normativos, que Durkheim finca sobre todo en el derecho positivo. De ese modo, la cohesión se realiza en el plano de las relaciones entre individuos pero reacciona y depende también de los imperativos y problemas que se generan desde los ámbitos estructurales (como la producción).

En este sentido, la cohesión refiere a la posibilidad de conflicto en las interacciones sociales $\mathrm{y}$, por ello, también a instancias que lo contengan o inhiban. Aunque el conflicto en las interacciones está en el centro del concepto de cohesión, algunos autores han mostrado que ese no deriva solo de imperativos estructurales ni se regula sólo por normas jurídicas sino de otro tipo, como las culturales, sociales o cívicas (Berger, 1999). Así, cohesión refiere a un espectro amplio de regulación normativa, o axiológica, de las interacciones entre individuos de modo que se produzcan dos efectos: que éstas se hagan posibles y se amplíen cada vez más entre distintos y, es obvio, se reduzca la posibilidad de conflicto a efecto de que se mantenga un cierto horizonte de estabilidad. La tolerancia, por ejemplo, coadyuva a tal fin.

En esa línea, si consideramos contextos relacionales y de experiencia del individuo, el efecto positivo de la cohesión social sobre el BS parecería evidente. Teóricamente nadie se sentiría satisfecho si sus relaciones fueran conflictivas o si estuvieran acotadas a un número predeterminado sin oportunidad de ampliarse. Los resultados de la investigación, sin embargo, son sorprendentes. Para el análisis de la cohesión social elaboramos tres variables compuestas: a) conflicto con familiares, 
amigos y vecinos; b) índice de fragmentación social; y c) posibilidades de extensión de contactos sociales.

Para el conflicto ${ }^{6}$, como indicamos, se tomaron en cuenta las relaciones con familiares y amigos de un lado, y con vecinos, de otro. De conformidad con cierta literatura, estas tres áreas de experiencia tienen enorme importancia para BS porque constituyen espacios de convivencia inmediatos para el individuo. Helliwell y Putnam (2004, p. 1.441) anotan para US y Canadá:

Frequent interactions with friends and neighbours are both associated with systematically higher assessments of subjetive well-being [...] frequent interactions with friends are even more important (especially in US) than those with neighbours and family, with family contact being slightly more important than that with neighbours.

En contraste, es notable que conflicto con familiares o amigos - o su ausencia no parezca tener ninguna influencia en el BS en ninguna de las dos ciudades. Pese a que el $76 \%$ de las personas que experimentan conflicto en sus relaciones se declara feliz en Chilpancingo (CHP) y el $80 \%$ en Monterrey (MTY), tanto las pruebas de independencia como la regresión mostraron nula asociación. El dato es relevante. El vínculo relativamente firme entre ausencia de conflicto y BS que teóricamente se ha establecido requeriría, ante ese dato, una explicación. Distintas investigaciones han mostrado que dinámicas no conflictivas en la familia se asocian con menor estrés, ausencia de depresión, buena comunicación, libertad en la expresión afectiva y por eso se les vincula con satisfacción marital (Olson y De Frain, 2000). No es desatinado presumir que entre amigos la "satisfacción fraterna" debería recoger algunas de esas características. Adicionalmente, de conformidad con la literatura, la familia se encuentra entre las variables más importantes para el BS y, con un peso menor pero igualmente sostenido, los amigos también lo son (Moyano y Ramos, 2007; García, Fuentes y Borrego, 2006; Florenzano y Dussaillant, 2011). Lo intrigante, entonces, es esto: ¿porqué, en las dos ciudades estudiadas, una mejor comunicación, expresividad y afectividad, así como los otros beneficios que acompañan a las relaciones no conflictivas, no son considerados como un factor en la apreciación de la propia felicidad?. Una explicación tentativa podría ser esta: en determinados contextos como los que nos ocupan, existe una cierta disociación entre felicidad y experiencias afectivas: la calidad de las relaciones en los ámbitos más cercanos, como la familia, no modulan la autopercepción del propio BS porque lo importante en esa valoración es la presencia o persistencia de las relaciones mismas, no la experiencia expresivo-afectiva específica con la que se realizan.

\footnotetext{
${ }^{6}$ Se preguntó qué tanto conflicto se tenía del 0 al 10 con familiares, amigos y vecinos, y se construyó una variable primero de tres categorías y luego de dos: conflicto/nada de conflicto. A partir de 7 se tomo como conflicto.
} 
La interacción entre vecinos presenta una variación que da muestra de la importancia de los contextos para el individuo. Mientras que en MTY no se registra efecto alguno en el BS, en CHP es un factor considerable y fuertemente asociado al BS. Dada la variable de referencia, tenemos que mientras más conflicto hay con los vecinos menos probable es que nos evaluemos como felices. Mantener relaciones armónicas con los vecinos incrementa, entre los felices, en 11 puntos porcentuales la probabilidad de serlo (Tabla 1 ).

La forma y peso de la participación en asociaciones nos ayudaría, tentativamente, a esclarecer la disparidad entre ambas ciudades. Medida como actividad anual, las personas en CHP participan (Gordon y Millán, 2009) en mayor grado en agrupaciones de cualquier tipo ( $47 \%$ contra $25 \%$ en MTY). También participan más en asociaciones cívicas, las cuales incluyen a las de vecinos ( $21 \%$ contra $6 \%$ ), y en organizaciones sociales o políticas ( $10 \%$ contra $2 \%)$. A esas diferencias, se agrega el rasgo de que cierto tipo de culturas clientelares como la que priva en CHP promueven más decididamente la asociación a nivel de barrios para articular grupos de vecinos con objeto de que funjan como apoyo político a cambio de gestoría de servicios. Es claro que la mayor participación en asociaciones genera espacios para los contactos vecinales en los que la ausencia de conflicto importa. Previsiblemente su peso para el BS será mayor si se percibe que, en contextos de asociación barrial, sostener malas relaciones con los vecinos eleva el costo personal ya que previsiblemente acarrea la exclusión de ciertos beneficios o servicios. $Y$ ese no parece ser el caso en MTY.

El efecto de la fragmentación social en el BS, y en especial en la felicidad, ha sido poco explorado, aunque tenemos algunas excepciones (Hoogle, 2012). Alternativamente a otras perspectivas, entendemos el concepto de fragmentación social en sentido amplio y no solo económico o ligado a ese tipo de desigualdad (Guardiola y García-Muñoz, 2009; Melgar y Rossi, 2011). 
Tabla 1

Chilpancingo: probabilidad de ser feliz (en porcentaje)

\begin{tabular}{|c|c|c|}
\hline & $\mathbf{P}$ & $1-\mathbf{P}$ \\
\hline Variable & (Feliz) & (Poco o nada feliz) \\
\hline \multicolumn{3}{|l|}{ Edad del entrevistado } \\
\hline Edad (20 años) & 87,6 & 12,4 \\
\hline Edad (30 años) & 82,3 & 17,7 \\
\hline Edad (40 años) & 78,9 & 21,1 \\
\hline Edad (50 años) & 78,5 & 21,5 \\
\hline Edad (60 años) & 81,4 & 18,6 \\
\hline Edad (70 años) & 86,5 & 13,5 \\
\hline \multicolumn{3}{|l|}{ Conflicto con vecinos } \\
\hline Nada de conflicto & 84,8 & 15,2 \\
\hline Conflicto & 73,5 & 26,5 \\
\hline \multicolumn{3}{|l|}{ Índice de fragmentación } \\
\hline Pocos o ningún amigo con preferencias diferentes & 84,5 & 15,5 \\
\hline Muchos amigos con preferencias diferentes & 73,6 & 26,4 \\
\hline \multicolumn{3}{|l|}{ Establecer nuevas relaciones } \\
\hline Difícil & 78,2 & 21,8 \\
\hline Fácil & 83,8 & 16,2 \\
\hline \multicolumn{3}{|l|}{ Confianza en la familia } \\
\hline Confía poco o nada & 71,4 & 28,6 \\
\hline Confía totalmente & 85,6 & 14,4 \\
\hline \multicolumn{3}{|l|}{ Personas se tratan como iguales } \\
\hline No se tratan como iguales & 81,2 & 18,8 \\
\hline Si se tratan como iguales & 86,9 & 13,1 \\
\hline \multicolumn{3}{|l|}{ Cooperación en proyecto colectivo } \\
\hline No coopera & 77,4 & 22,6 \\
\hline Sí coopera & 83,9 & 16,1 \\
\hline
\end{tabular}




\begin{tabular}{|c|c|c|}
\hline & $\mathbf{P}$ & $1-\mathbf{P}$ \\
\hline Variable & (Feliz) & (Poco o nada feliz) \\
\hline \multicolumn{3}{|l|}{ Interés asuntos políticos del país } \\
\hline Muy interesado & 91,7 & 8,3 \\
\hline Interesado & 90,1 & 9,9 \\
\hline Más o menos interesado (indiferente) & 76,6 & 23,4 \\
\hline Poco interesado & 79,7 & 20,3 \\
\hline Nada interesado & 79,4 & 20,6 \\
\hline \multicolumn{3}{|l|}{ Gobierno considera opinión gente } \\
\hline Mucho o algo & 89,3 & 10,7 \\
\hline Indiferente & 65,7 & 34,3 \\
\hline Poco o nada & 81,1 & 18,9 \\
\hline \multicolumn{3}{|l|}{ Satisfacción lugar donde vive } \\
\hline Bueno & 85,7 & 14,3 \\
\hline Regular & 78,7 & 21,3 \\
\hline Malo & 68,5 & 31,5 \\
\hline \multicolumn{3}{|l|}{ Ingresos del hogar } \\
\hline Hasta dos salarios mínimos & 75,3 & 24,7 \\
\hline Más de dos y menos de cuatro s.m. & 84,8 & 15,2 \\
\hline De cuatro a seis s.m. & 91,9 & 8,1 \\
\hline Más de seis s.m. & 92,7 & 7,3 \\
\hline
\end{tabular}

Fuente: Encuesta propia. 
Tabla 2

Monterrey: probabilidad de ser feliz (en porcentaje)

\begin{tabular}{|c|c|c|}
\hline & $\mathbf{P}$ & $1-P$ \\
\hline Variable & (Feliz) & (Poco o nada feliz) \\
\hline \multicolumn{3}{|l|}{ Edad del entrevistado } \\
\hline 39 o menos años & 88,3 & 11,7 \\
\hline Más de 39 años & 83,9 & 16,1 \\
\hline \multicolumn{3}{|l|}{ Confianza en la familia } \\
\hline Confía poco o nada & 79,9 & 20,1 \\
\hline Confía totalmente & 86,9 & 13,1 \\
\hline \multicolumn{3}{|l|}{ Personas se tratan como iguales } \\
\hline No se tratan como iguales & 84,7 & 15,3 \\
\hline Si se tratan como iguales & 89,4 & 10,6 \\
\hline \multicolumn{3}{|l|}{ Í ndice de contactos con amigos } \\
\hline No realiza actividades con amigos & 79,1 & 20,9 \\
\hline Si realiza al menos una actividad & 87,4 & 12,6 \\
\hline \multicolumn{3}{|l|}{ Respetan sus derechos } \\
\hline Sí & 86,2 & 13,8 \\
\hline No & 80,6 & 19,4 \\
\hline Algunos sí otros no & 89,9 & 10,1 \\
\hline \multicolumn{3}{|l|}{ Satisfacción lugar donde vive } \\
\hline Bueno & 90,0 & 10,0 \\
\hline Regular & 76,0 & 24,0 \\
\hline Malo & 67,5 & \\
\hline \multicolumn{3}{|l|}{ Ingresos del hogar } \\
\hline Hasta dos salarios mínimos & 80,7 & 19,3 \\
\hline Más de dos y menos de cuatro s.m. & 82,4 & 17,6 \\
\hline De cuatro a seis s.m. & 90,6 & 9,4 \\
\hline Más de seis s.m. & 90,8 & 9,2 \\
\hline
\end{tabular}

Fuente: Encuesta propia. 
Para el análisis de la fragmentación se constituyó un Índice de Fragmentación Social (IFS) a partir de siete enunciados que indican la presencia de relaciones, o no, entre personas distintas. Distintas por su capacidad económica, educación, religión, pertenencia generacional y por ideología ${ }^{7}$. Al elaborar el IFS asumimos el presupuesto de que individuos que se desenvuelven en contextos sociales que integran y vinculan a distintas redes, pertenecen también a comunidades plurales que han incrementado su propia conectividad interna. La conectividad de una sociedad está ligada a la formación de puentes o brigdes (Granovetter, 1973) y al incremento del capital social por esa vía (Putnam y Goss, 2002). El capital social genera bonding o vínculos cuando conecta a gente similar a partir de alguna característica común (edad, genero, consanguinidad, sector social) y forma puentes o bridges cuando las redes incorporan gente diversa entre sí (Millán y Gordon, 2004; Putnam y Goss, 2002).

De manera todavía más sorprendente que el conflicto con vecinos, el IFS arroja resultados contrastantes que niegan la relación directa y mecánica entre no fragmentación social y bienestar. Mientras que en MTY el índice no tiene efecto en el BS, en CHP tiene una asociación significativa pero de signo negativo (Cuadro 1 ). Dado que la categoría de referencia es no experimentar fragmentación, nos encontramos, en efecto, frente al desconcertante caso de que mientras más fragmentación hay más posibilidades de ser feliz existen. Este "contra-sentido" puede ser explicado. Hoogle (2012), en un estudio sobre cohesión social en Bélgica, parte de un dato paradójico de los países desarrollados de Occidente: mientras que ciertos estudios muestran que los niveles de BS suben, se mantienen estables, o no decrecen significativamente, la desafección con las sociedades o de descontento con su desempeño se incrementan. En particular, nos dice Hoogle, se aprecia un sentido de pérdida de comunidad. Así, altos niveles de BS "can be combined with a negative outlook on the society one lives in" (2012, p. 19). Esas dos variables, BS y evaluación de la comunidad de referencia, son dos conceptos distintos por lo que medir una no implica necesariamente evaluar la otra. Es factible asociarlas, sin embargo, a partir de un tercer factor.

El etnocentrismo es para Hoogle ese tercer factor. Lo describe como "negative attitud to the outsider groups, that are seen as a potential threat to the culturally or economically dominant position on our own insider group" (Hoogle, p. 21). El etnocentrismo muestra una relación positiva con el BS pero una negativa con la evaluación de la sociedad en términos de comunidad (p. 22-25). La idea del reforzamiento de los propios grupos y el rechazo de los "externos" no occidentales,

\footnotetext{
${ }^{7}$ Se hicieron siete preguntas a propósito de si se tenía amigos distintos, y cuantos, por nivel económico, educación, religión, ideas políticas y edad. La escala tuvo cuatro valores (todos, la mayoría, algunos, ninguno). Con la recodificación a las categorías "Todas o la mayoría" se le asignó el valor 1 y a "Algunas o ninguna" el valor 0 . Aquellos entrevistados que obtuvieron 1 en tres o más de los enunciados (es decir que tienen 3 o más amigos de diferente) no experimentan la existencia de un ambiente fragmentado mientras que aquellos que obtuvieron 1 en dos o menos de los enunciados si lo perciben.
} 
implícita en el razonamiento, hacen que la argumentación de Hoogle esté de hecho formulada en términos de fragmentación social. El grado de integración de redes o grupos similares en correspondencia con la desintegración entre ellos modula la intensidad de la fragmentación social. La paradoja de los resultados toma así sentido. Sobre todo si sustituimos etnocentrismo por la presencia de una comunidad regida por "lazos fuertes" o bondings considerablemente encapsulados.

En efecto, en CHP el IFS nos indica que se trata de una comunidad extremadamente segmentada. Esa condición describe, en efecto, una sociedad fincada en lazos fuertes. Estos lazos tienen en la familia y en las amistades cercanas sus referentes más claros. Un estudio (Ellison, 1991) basado en la National Survey of Black American (NBSA) muestra que los vínculos de parentesco están asociados positivamente con el BS, si bien eso decrece con la edad. La solidez de los vínculos está determinada por la frecuencia de los contactos, indicador como sabemos de lazos fuertes. Sin embargo, según el mismo estudio, la ampliación de esos contactos hacia la familia extensa tiene efectos negativos en la satisfacción de vida. Se podría afirmar que la fragmentación va teniendo distintos efectos en el BS de conformidad con la estructura de vinculación entre grupos y redes y en función de que la presencia predominante de lazos fuertes. En esa lógica, sería comprensible que para ciertos individuos mientras más se desempeñan en ambientes altamente integrados u homogéneos, más alto evalúan su BS. De hecho, esa es la tesis que subyace en la idea de etnocentrismo de Hoogle. El "refugiarse" en ámbitos o redes altamente integradas indica la lógica "comunitaria" que rige a CHP. Los siguientes datos parecen comprobarlo: la probabilidad de ser feliz al mantener relaciones casi exclusivamente con personas similares es de $84 \%$ contra un $74 \%$ de serlo cuando la mayoría de los vínculos son con individuos diferentes (Tabla 1). Es una diferencia de 10 puntos.

El grupo de argumentaciones anteriores, plausible para CHP, plantea otro problema: ¿por qué el IFS es indiferente para la felicidad en MTY? Es difícil responder pero podemos intentar una respuesta tentativa. Como se verá enseguida, aunque la confianza en la familia es relevante para ser feliz en ambas ciudades, la sociabilidad lo es sólo en MTY. Eso significa que los individuos de esa comunidad se "refugian" también en los amigos y no sólo en la familia como ocurre principalmente en CHP.

En conjunto, el cuadro del IFS nos lleva a una conclusión importante. Si la tesis de fondo sobre lazos fuertes y conectividad que se deriva del capital social (Putnam, Leonardi e Nanetti, 1994) es correcta, deberíamos entonces asumir que la forma e intensidad de la fragmentación social afecta el modo en que los vínculos sociales influyen en el BS.

La última variable que exploramos como parte de la cohesión social es la facilidad para hacer nuevas relaciones. En CHP, el BS está discreta pero significativamente asociado a la experiencia de vivir en una comunidad que facilita el 
incremento de sus vínculos sociales. La diferencia con quienes creen que es difícil ampliar los propios círculos es de 6 puntos porcentuales (Tabla 1). Es notable que en MTY la variable no resultó relevante para el modelo de regresión logística, pero en términos de las pruebas de asociación e independencia mostró estar significativamente vinculada con la felicidad. Quizás no sea apresurado suponer que en una comunidad donde las relaciones informales están centradas sobre todo en ámbitos familiares, el percibir que es posible ampliar la propia red con relativa facilidad pese más que en una comunidad en la que, por cualquier razón, la sociabilidad está más focalizada en los amigos. De nuevo, esto remitiría a la tesis de que la forma de la fragmentación social modula el impacto de los vínculos sociales en el BS.

\section{Capital social}

Las alusiones hechas a ese tipo de capital en la sección anterior han ayudado a comprender el comportamiento del IFS pero no hemos hasta aquí sugerido ninguna variable para medirlo. El soporte fue de tipo conceptual. En esta sección pondremos a prueba su relación con el BS. En términos generales, hay un amplio acuerdo en la literatura especializada sobre la sólida y positiva asociación entre ambos (Helliwell y Putnam, 2004). Por la complejidad de su composición y la natural extensión de los temas con los que se correlaciona, el concepto admite un grado considerable de flexibilidad.

Disciplinariamente, son tres las principales perspectivas. La conocida definición de Coleman lo establece como una cualidad inscrita en la estructura de las relaciones o interacciones y, en esa lógica, como un recurso que facilita la coordinación de acciones o la cooperación (1990, p. 302). Esa cualidad se da por normas que estructuran obligaciones y expectativas recíprocas entre los actuantes en la interacción, particularmente confianza y reciprocidad; por relaciones de autoridad derivadas de factores institucionales o carismáticos; o por la forma en que se logra la "clausura" de una red de relaciones porque ella genera cohesión al reforzar normas y expectativas comunes (Coleman, 1988 y 1990). En línea con Coleman, Putnam (2000) acentúa la confianza y las normas de reciprocidad de modo que subraya que la calidad de los vínculos determina el capital social. Para Putnam (2000), las redes sociales "densas" tienen un papel fundamental en su formación de capital social. Las redes interesan porque son "escuelas" de aprendizaje de normas de reciprocidad y generadoras de confianza interpersonal. Esas virtudes se acentúan en las redes cívicas y voluntarias porque se articulan mediante relaciones de tipo horizontal y, justo por eso, inducen a la reciprocidad y a una más lograda formación de capital social. Las redes verticales (clientelares), en cambio, mantienen obligaciones recíprocas pero de manera asimétricas: los vínculos verticales socavan la confianza y el carácter generalizado de la 
reciprocidad (Putnam, 2000, cap. 6).

Brevemente, Ostrom y Ahn (2003) asumen como capital social un amplio rango de aspectos que favorecen la cooperación voluntaria para atender problemas comunes. De ahí también que lo conciba como encarnado en diversas "formas sociales" más que como un concepto que articula distintos componentes. Entre ellas, distingue tres: a) confianza y normas de reciprocidad; b) redes y formas de participación civil; y c) reglas o instituciones formales e informales. Para esta perspectiva, en función de la relación entre las dos últimas formas de capital social, se modula el efecto positivo de la confianza y reciprocidad en el éxito de las acciones colectivas. De ese modo, la confianza y la reciprocidad no derivan solo, por así decir, de una interacción repetida sino que obedece también a contextos institucionales más generales.

En conjunto, las distintas nociones de capital social reseñadas involucran aspectos como la cooperación social, la confianza, normas de reciprocidad, participación cívica, instituciones, entre otras. Para efectos de este artículo, identificaremos esas concepciones con una idea "amplia" de capital social. En ella, es Ostrom la que más ha declinado acentuar la dimensión interactiva que está implícita en aquel concepto y lo hace a favor del contexto que modulan las reglas e instituciones particulares de un ambiente social. Este aspecto reviste vital importancia. Si por el contrario asumimos una perspectiva centrada en las relaciones interpersonales, es fácil subsumir al capital social en la cantidad y calidad de las relaciones interpersonales y, en esa línea, identificar los vínculos sociales y los llamados bienes relacionales como parte de él. Llamaremos a esta perspectiva "restringida". En un conocido artículo, Helliwell y Putnam (2004, p. 1.437) identifican al capital social, a fin de determinar su asociación con el BS, básicamente como redes de diverso tipo: matrimonio, "family members, close friends and confidants, friendly neighbours and supportive coworkers". De ese modo, se focaliza en un stock de conexiones cargadas de acompañamiento, reconocimiento mutuo, afectividad. Se trata de interacciones regidas por una marcada lógica de sociabilidad. De ahí, su posible equiparación a los bienes relacionales (Becchetti, Pelloni y Rossetti, 2008).

Dentro de la versión "amplia", analizaremos aquí cuatro variables: confianza, reciprocidad, participación en asociaciones y cooperación. Dentro de la "restringida" tomaremos dos variables: confianza en la familia y sociabilidad. En términos de la literatura internacional, como quiera que se mida, el nivel de asociación atribuido al capital social con el BS es consistente. Adelantamos que en esta investigación el resultado es, para las dos ciudades, más contrastante. Tal contraste está determinado por la distinción apenas hecha: la concepción "amplia" no tiene el peso de la "restringida" pero en ambas los resultados son heterogéneos y no están siempre en línea con los resultados internacionales.

Para mostrar el contraste y heterogeneidad en los resultados, es conveniente 
analizar conjuntamente confianza y reciprocidad. Como se sabe, es la confianza el elemento de mayor relevancia para el capital social y el que conlleva mayores consecuencias notables para las sociedades (North, 1993; Fukuyama, 1995; Torsvik, 2000). Sin embargo, teóricamente confianza y reciprocidad trabajan hermanadamente. Para quien sostiene una relación, si alguien no es recíproco tampoco es confiable. Putnam distingue entre reciprocidad específica y general (Putnam, 2000). La primera se conforma mediante intercambios inmediatos, cara a cara o en el seno de una red cerrada. El trueque implica "valores" más o menos equivalentes: un favor por otro, un pequeño préstamo monetario por un servicio cuyo costo es semejante. La generalizada, en cambio, tiende a equilibrar los valores del intercambio en el tiempo, no en cada operación concreta, y normalmente se da de manera indirecta, más allá de una interacción inmediata entre los presentes: por ejemplo, cuando un ciudadano no estaciona su coche frente a mi cochera porque sabe que yo respetaré su lugar en la fila del cine (Millán y Gordon, 2004). Cuando eso ocurre, hablamos en efecto de una norma de reciprocidad generalizada. Una reciprocidad generalizada debería corresponder con una confianza general y, por contra-lógica, una acotada con una confianza encapsulada en grupos o redes ${ }^{8}$ (Fukuyama, 1995). Como veremos, esa coherencia teórica no se cumple pulcramente en el análisis de los casos.

Dada la discusión sobre IFS, arriba sostenida, no deberían asombrarnos el resultado de la relación entre confianza y BS. En ninguno de los casos, la relación es significativa. En contraste, la reciprocidad está en ambas ciudades asociada positivamente a la felicidad (Cuadros 1 y 2 ) aunque no con mucha fuerza. La probabilidad de ser feliz si se aprecia reciprocidad es, en CHP, de $87 \%$ contra un $81 \%$ si no se percibe, seis puntos porcentuales de diferencia (Tabla 1). En MTY es de cinco puntos: $90 \%$ contra $85 \%$. (Tabla 2). Pese a lo acotado de los rangos, la ausencia de relación con la confianza requiere una explicación.

La pregunta sobre si las personas se tratan como iguales, se utilizó para medir la reciprocidad. El "trato igualitario" es con toda evidencia una regla o norma de reciprocidad con dimensiones sociales, cívicas y políticas. Implica derechos y dimensiones de respeto y dignidad de las personas. El tema, entonces, es dilucidar cómo se da la reciprocidad en contextos de fragmentación social acentuados y sin confianza. En primer lugar deberíamos considerar que "la calidad de una regla, una institución o un estatuto como forma de capital social depende no sólo de su contenido sino, más críticamente, de cómo se aplica en la realidad" (Ostrom y Ahn, 2003, p. 184). Y al parecer, en CHP, la reciprocidad expresa sobre todo un código de cortesía con que se conducen las interacciones entre no cercanos en el espacio público. En segundo lugar, se debe recordar que, en ciertas circunstancias, la reciprocidad como un estricto parámetro de interacción en sí es, en términos de teoría de juegos, un equilibrio que no

\footnotetext{
${ }^{8}$ Es en esta línea que se habla del "lado oscuro" del capital (Portes e Landolt, 1996).
} 
incentiva coordinar acciones ni confianza (Ahn, 2000; Kollock, 1998). Particularmente cuando se trata de una reciprocidad específica y no generalizada.

Además del IFS, otra evidencia de que no se trata (para ninguna de las ciudades) de una reciprocidad generalizada se encuentra en el comportamiento de la variable participación en asociaciones cívico-voluntarias. Esa participación es un elemento central del capital social porque se asume que las redes en ellas conformadas funcionan como escuelas de confianza y reciprocidad (Millán y Gordon, 2004; Ostrom y Ahn, 2003; Putnam, 2000). Para perspectivas como Putnam, las asociaciones impulsan la reciprocidad generalizada.

Al cruzar participación en al menos una asociación y felicidad nos encontramos con que no hay vinculación en ninguna de las ciudades. El resultado es sorprendente porque se consideró un espectro de 20 tipos de asociaciones: de religiosas a jubilados. El resultado contradice los hallazgos internacionales. En un trabajo basado en tres secuencias de la Encuesta Mundial de Valores, Helliwell (2002, p. 13-14) encuentra que Ios

individuals who are involved in more voluntary associations report higher average satisfaction with their lives [...] someone with additional voluntary group membership [...] has a well-being that is higher by 0.05 , about tenth as much as marriage.

Es un vínculo fuerte en consecuencia. Varios estudios han subrayado el hallazgo de que la participación en asociaciones cívicas no solo está positivamente ligada al BS sino a un claro sentido de comunidad, reciprocidad y confianza (Thoits, Hewitt y Volunteer, 2001).

La coherencia anterior entre variables no se da para el caso de nuestras dos ciudades. Si las asociaciones cívicas no son fuentes de confianza generalizada no pueden estar ligadas a una reciprocidad generalizada. En consecuencia, es muy probable que la reciprocidad que prive entre esas asociaciones se acote a grupos específicos, subredes, del mismo modo que lo hace la confianza. Axelrod (1984) ha demostrado que cuando agentes en ámbitos de reciprocidad probable se deciden por estrategias de Tic-for-Tac (parecida a la reciprocidad específica) el intercambio se queda en grupos pequeños o redes cerradas. La idea de que esa lógica opera en las dos ciudades estudiadas, se refuerza al recordar que Brehm y Rahn (1997) han constatado que no siempre, ni automáticamente, existe un efecto de generación de confianza y reciprocidad a través de la participación en asociaciones. Stolle (2011), además, mostró que cuando se generan es probable que queden concentradas en grupos sin que se generalicen más allá. Así, no es del todo preciso presumir que toda red cívica implícitamente "exporta" o "expande" la confianza social o que esa se distribuye igual entre sus miembros. Esta argumentación es consistente con la idea, sostenida aquí, de que el efecto de los componentes del capital social en el BS se ve modulado por los 
niveles y formas de la integración sociopolítica y, en particular, por los modos de la cohesión social.

Es prácticamente inexistente el análisis de los vínculos entre cooperación social y BS. Se encuentran implícitos en los análisis del capital social pero, dada la importancia de los contextos, es prudente distinguirla. De hecho, el comportamiento de esta variable respecto de la confianza y en referencia a la felicidad es, de nuevo, contrastante. La pregunta que se midió fue esta: "si un proyecto de la colonia no lo beneficiara a usted directamente pero si a otros vecinos ¿colaboraría o no para realizar el proyecto?". Es importante notar que la pregunta se ubica en el ámbito de lo vecinal. En CHP, cooperar con otros está positivamente ligado al BS (Cuadro 1). La probabilidad de ser feliz si se coopera es de $84 \%$ contra un $77 \%$ si no se hace (Tabla 1 ). La diferencia es ilustrativa.

En contraste, MTY no reporta alguna asociación significativa. ¿En qué podría consistir la diferencia entre ambas ciudades? Una respuesta probable es esta: la asociación positiva de la cooperación está en línea con la importancia que, en CHP, tiene el conflicto con vecinos en la determinación del BS y en correspondencia con la indiferencia que en MTY muestra esa variable. Contextualmente, entonces, el peso de los vecinos es mayor en una ciudad que en otra. La relación vecinal en CHP se encuentra barrialmente más cohesionada y en ese sentido cooperar con los vecinos es también una estrategia para evitar posibles conflictos. Pero ¿por qué esa cohesión en una comunidad que, en general, está altamente fragmentada? Recordemos que el grado de modalidad clientelar de la intervención estatal es más acentuada en CHP y que ese clientelismo tiene costos de exclusión. De nuevo, la diferencia entre ciudades tiene relevancia.

La concepción "restringida" de capital social la hemos identificado con los Ilamados bienes relacionales. En los últimos, el estudio de los bienes relacionales se ha expandido porque muestran estar sólidamente asociados al BS (Becchetti, Giachin y Pelloni, 2009). Como el capital social, la idea de los bienes relacionales partió del hecho de que incluso en los mercados la gente se comunica, se encuentra, mantiene interacciones y éstas son importantes para aquellos. No extraña, entonces, que el concepto original surgió al relevar dimensiones de cierta sociabilidad implicada en ámbitos racionales como en la economía o la participación política, en apariencia altamente instrumentales. Se les entiende como un tipo particular de local public goods (Gui, 1987; Uhlaner, 1989). Son públicos porque sólo son "disfrutables" en conjunto y no de forma aislada; son "local public" porque su consumo se realiza por un grupo delimitado en zonas informales o profesionalizadas. Son específicos porque tienen una estructura no-rival: su naturaleza es ser compartidos (Becchetti, Pelloni y Rossetti, 2008).

En primer lugar, son "bienes" porque producen una cierta utilidad de goce y 
afectividad (Bartolini, 2012). En segundo lugar, las relaciones individuales no se organizan como una red homogénea y universal (hay hoyos estructurales) sino como redes delimitadas, acotadas. En tercer lugar, las relaciones pueden tener una estructura no-rival porque ninguna es igual a otra - y por ello son específicas. Sin embargo, en otro sentido, las relaciones sí están en grado de rivalizar y excluir según ámbitos de experiencia: es difícil mantener dos amores simultáneos, al menos más que dos amigos, y éstos pueden rivalizar, en tiempo y atención, con la familia. En cuarto y último lugar, las relaciones pueden agotarse. Si asumimos estas características, los relacionales se asemejan a los bienes comunes $y$, sobre todo, a los de club. Esta precisión es importante para lo que sigue en términos de resultados.

Por esas y otras consideraciones, algunos autores, en el tratamiento de los bienes relacionales, han acentuado la diferencia entre las dimensiones instrumental y afectivo/expresivo, más que el tipo de bien. $Y$ tal enfoque da, a mi juicio, más precisión que el de local goods. Becchetti, Pelloni y Rossetti (2008, pp. 1-4) entienden por bienes relacionales "affective/expressive, no instrumental, side of interpersonal relationships". Y agregan:

relational goods include companionship, emotional support, social approval, solidarity [...]. These goods are, on a smaller scale, produced by family relationships or friendships and, on a larger scale, in many kinds of social events (club or association meetings, live sport events).

Es decir, la afectividad que se enquista en ciertas pautas de sociabilidad. Para analizar los bienes relacionales, hemos tomado dos variables: confianza en la familia y sociabilidad con amigos.

Contrariamente a la interpersonal, la confianza en la familia es un factor determinante de la felicidad en ambas ciudades (Cuadros 1 y 2). En particular, en CHP representa una de las variables más significativas del modelo mientras su peso es más ligero en MTY. Confiar en la familia eleva sustantivamente la probabilidad de ser feliz. La probabilidad, para CHP (Tabla 1), de que una persona que confía manifieste un alto BS es de $86 \%$ contra un $71 \%$ de quien no mantiene relaciones confiables con sus parientes. Es una diferencia enorme ( 15 puntos porcentuales). En MTY, la diferencia es de 7 puntos, igualmente significativa pero con menor peso en la predicción (Tabla 2). Dado que ambas diferencias son altas, ilustran plenamente el papel que juega la familia en el BS. También confirman que los lazos en las familias de CHP son más estrechos que los de MTY.

Para la variable sociabilidad se creó un índice de contactos con amigos (ICA) con tres enunciados, referidos a los tres meses últimos de actividad: participación en juegos (de mesa, cartas etc.); visita a la casa de alguien o de amigos a la propia casa; reunirse con ellos fuera. A los que realizaron al menos una actividad se les asigno el valor $1=\mathrm{si}$, a los que no hicieron ninguna, $0=$ no. Como se aprecia el ICA no implica una actividad 
particularmente intensa, no obstante, su resultado es sorprendente. En Monterrey la relación con la felicidad es fuerte y positiva (Cuadro 2). La probabilidad de reportar un BS alto si se tiene contacto con amigos es $87 \%$ contra un $79 \%$, una diferencia de 8 puntos porcentuales (Tabla 2). En CHP, sin embargo, aunque hay una relación significativa en la pruebas de asociación no tiene peso en el modelo. Allí, tener contacto con amigos es una variable que no pesa en la determinación BS. Este resultado es consistente con el llamado "familismo" y la consiguiente tesis de los lazos fuertes expresada en la argumentación sobre el IFS. Por lo tanto, es también consistente con la tesis de que la intensidad y forma de la fragmentación tiene efecto en el tipo de variables que se asocian al BS, en este caso, en los bienes relacionales.

\section{Ciudadanía y gobierno}

Pese a que las asociaciones entre actividades o interés cívico-político y BS no tienen muchos estudios, en general se admite un vínculo positivo y virtuoso entre ambos. En línea teórica el vínculo tiene sentido de inmediato. Es indudable que el desempeño de gobiernos e instituciones públicas refiere también a una cultura política cuyas normas en los contextos particularizados de las personas, en las oportunidades que ellos perciben y en los comportamientos que ven como adecuados. Ott (2010), en un estudio comparado de 131 países de distinto calibre de desarrollo, muestra que hay una enorme diferencia entre el promedio más alto y el más bajo en una escala de 10 (Togo, 3; Denmark, 8). Su conclusión es que las diferencias de felicidad se relacionan con la calidad de la intervención del gobierno.

Guven (2009, p. 3), en un estudio basado en la German Socio-Economic Panel (GSOEP), encuentra que "happier people are found to have higher respect for law and order [...] have a higher desire to vote". En otra investigación realizada en Latino América, Weitz-Shapiro y Winters (2008) descubren una relación significativa entre participación política y el BS, en el sentido de que los felices votan más. En un análisis realizado sobre MTY, García, Fuentes y Borrego (2006) muestran que los valores influyen en el BS, entre ellos el interesarse en la política o en alguna causa social como el medio ambiente. Para medir el efecto de las relaciones políticas en el BS hemos considerado tres variables: participación electoral; interés en los asuntos políticos y respecto de los derechos.

En ninguna de las dos ciudades, la participación electoral, entendida como haber votado en las últimas elecciones, tiene un efecto en el BS. El interés por influir en el sentido u orientación del gobierno resultó ser una variable absolutamente indiferente para la felicidad. En contraste, el interés por los asuntos públicos nacionales muestra ser un factor que si influye en la felicidad de las personas pero sólo en CHP (Cuadro 1 ). La respuesta a la pregunta admitió cinco valores de los cuales tomamos como variable 
de referencia "muy interesado". Los valores fueron: interesado; más o menos interesado; poco interesado y nada interesado. Mientras menos indiferencia se muestra hacia los asuntos políticos de la nación, más aumenta la probabilidad de ser feliz: de nada interesado a muy interesado el rango va de $79 \%$ a $92 \%$ (Tabla 1 ). Es decir, pasar de nada a muy interesado eleva 13 puntos porcentuales la probabilidad de un BS alto.

El interés por asuntos públicos es indicador de compromiso cívico. El resultado contrasta con la nula influencia de la participación electoral. En línea con lo sostenido, una reflexión sería esta: en contextos de escasa alternancia electoral, incluso local, pero de fuerte presencia clientelar, el voto resulta irrelevante pero no los "asuntos públicos". Se trata un compromiso "no cívico" para decirlo en términos de Putnam, regido por relación o redes jerárquicas y clientelares (Putnam, 2000, cap. 5). En cualquier caso, es importante hacer notar que el compromiso cívico se encuentra más disminuido en MTY, ciudad menos clientelar, con menor intervención pública y con una cultura que da mayor peso al individuo. Este rasgo se expresa en la siguiente variable.

El hecho de vivir en una ciudad donde se respeten los derechos de los individuos no es una variable que afecta el BS de los ciudadanos de CHP. Una variedad enorme de investigaciones han mostrado cómo las lógicas corporativas y clientelares que privaron masivamente en el país - y en regiones como CHP - tienen a privilegiar derechos de organizaciones y a disminuir la importancia de los individuales (Millan, 2008). Es algo parecido a lo que, en términos de Putnam (2000), es una comunidad no cívica que él identifica con las sociedades del sur de Italia. En contraste, en MTY resulta ser una variable asociada al BS aunque con cierta debilidad (Tabla 2). El percibir que sí se respetan los derechos y ser feliz tiene una probabilidad de $86 \%$ contra un $80 \%$ si se cree que no se respetan (Tabla 2); son 6 puntos de diferencia. Persiste, entonces, un ligero pero claro acento en las condiciones en las que la propia individualidad se desempeña, las cuales contrastan con la cultura de CHP. Son dos contextos diferentes y coinciden con dos modalidades de cohesión social. El comportamiento de las tres variables anteriores parece indicar que la cultura política afecta la relación entre BS y ciertas variables en que forman parte de la integración sociopolítica. Lo contextual es de nuevo relevante.

\section{Calidad de vida}

Para concluir, hemos analizado dos variables de integración socioeconómica que, en términos muy generales, son indicativas de la calidad de vida de los individuos: las condiciones de habitabilidad e ingreso. Ambas variables dan prueba de que un cierto grado de calidad de vida es necesario para lograr el BS.

La importancia del contexto particularizado, aquel que el individuo experimenta en relación con su entorno físico y social, se muestra con toda claridad en esta 
dimensión. La calidad de vida y BS están persistentemente asociados (Lora, 2008). Veenhoven (2000) distingue entre dos dimensiones de esa calidad: la que se refiere a la habitabilidad del ambiente y la que tiene que ver con la habilidad para la vida de la persona que se desenvuelve en ese ambiente. Para esta variable nos interesa la primera dimensión. Yamamoto y Feijoo (2007), en un estudio realizado en Perú, encuentran que las condiciones de habitabilidad del lugar donde se vive tienen un efecto determinante sobre el bienestar. Es el mismo resultado que encontramos en nuestra investigación para las dos ciudades, si bien es más fuerte en MTY.

Para medir la variable se solicitó una auto-evaluación del lugar donde se vivía a partir de tres valores: bueno, regular y malo. La satisfacción con el lugar donde se vive está fuerte y significativamente asociada al BS en las dos ciudades (Cuadros 1 y 2). La probabilidad de ser feliz disminuye considerablemente si se aprecia que el lugar donde se vive no es bueno. Las probabilidades para CHP son: bueno, $86 \%$; regular, $79 \%$ y malo, 69\% (Tabla 1). En ese orden para MTY, son: 90\%, 76\% y 68\% (Tabla 2). En el primer caso tenemos una diferencia de 16 puntos porcentuales y condiciones de habitabilidad son, en efecto, una de las variables de mayor peso social en la determinación del BS.

De conformidad con cierta literatura, el ingreso muestra una fuerte asociación positiva con el BS. Sin embargo, también de acuerdo con cierta literatura, se estabiliza o su utilidad decrece en términos de bienestar. Aspecto conocido ya desde la paradoja de Easterlin (1974). La variable se midió como ingreso familiar y en "salarios mínimos". En ambas ciudades, la probabilidad de ser feliz se incrementa sostenidamente a medida que crece el ingreso (Cuadros 1 y 2). Para CHP: hasta dos salarios, 75\%; de dos a cuatro, 85\%; de cuatro a seis, 92\%; y de más de seis salarios, 93\% (Tabla 1). Para MYT, respectivamente: $81 \% ; 82 \% ; 91 \%$ y $91 \%$ (Tabla 2 ). Como se aprecia, en la primera ciudad conforme se incrementa el salario se reduce la diferencia entre los ingresos inmediatos, en MYT se aprecia la misma lógica pero con un "salto" claro: entre cuatro y seis salarios mínimos. Los resultados muestran que una base mínima económica es absolutamente indispensable para el BS. Y aunque el ingreso, a partir de cierto monto, pierda eficacia, nada indica que produzca más infelicidad.

\section{Consideraciones finales}

a) El análisis comparado entre las dos ciudades dio suficiente evidencia de que la integración sociopolítica es un factor importante en la definición y peso de las variables relacionadas con el BS. Sus formas pueden aclarar en mucho la heterogeneidad del comportamiento de esas variables. Los contextos efectivamente importan. Es notable que en la comparación entre las dos ciudades solo las variables confianza en la familia, reciprocidad, habitabilidad e ingreso tengan significación en 
ambas. En cambio, conflicto con vecinos, fragmentación social, facilidad para hacer relaciones, cooperación, sociabilidad, interés en asuntos públicos y derechos se comportan de modo distinto en cada una.

b) En el marco de la contextualización del BS, la cohesión social se muestra como una de las variables de mayor peso e importancia para explicar sus determinaciones. En particular, la forma en que se da, o no, la fragmentación social. De acuerdo con los resultados mostrados, se pueden establecer dos proposiciones vinculadas. De un lado parece ser claro que en ámbitos sociales muy agregados (en la familia o círculos cercanos) no se incorpora la percepción que se tiene sobre la calidad de las relaciones del grupo de referencia como un factor clave en la definición del BS. Lo que importa es la mera pertenencia al grupo y en consecuencia se subvaloran los aspectos expresivo-afectivos en la determinación del propio bienestar. Como vimos, la causa de ese balance es una cohesión social fincada sobresalientemente en lazos fuertes. De otro lado, ellos también explican el peso de la fragmentación social en el BS. Mientras más fuertes son los lazos que rigen la cohesión social de modo preeminente, más fragmentada está una sociedad pero su peso en el BS parece ser menor tal y como ocurre en CHP. Es decir, si la cohesión social está estructurada mediante lazos fuertes la fragmentada vinculación entre grupos y redes no afecta al BS. Este se refuerza en los grupos y redes de referencia. Como hemos visto, mientras más acusada es la fragmentación por lazos fuertes, menos importancia tiene la sociabilidad en el BS como en CHP. En contraste, mientras más relajada es la cohesión social más cuenta la sociabilidad, como en MTY.

c) La forma de integración también afecta el peso que los componentes del capital social, en su versión amplia y restringida, tienen en el BS como se muestra en las dos ciudades. Aunque en MTY las interacciones con los otros abarcan también la amistad, tienden como en CHP a ser un cuerpo de relaciones acotadas en ámbitos muy cercanos. De ahí se desprende, por ejemplo, que la reciprocidad que importa sea de tipo específico y no esté ligada a formas de compromiso cívico como establece la teoría y se verifica en otros países.

d) La forma de integración sociopolítica también afecta la coherencia teórica entre las variables de capital social y su peso en el BS. Según los resultados, se tienen enormes contrasentidos. Por ejemplo, en CHP si importa el conflicto con los vecinos y el cooperar con ellos para el BS, pero eso no es producto de un compromiso cívico de acuerdo con el capital social, es el resultado de una presión que se ejerce por pertenecer a una sociedad fincada en un corporativismo fuertemente afirmado. De ahí, que el no tener derechos no afecte el BS, como si lo hace en MTY. Es decir, las formas 
en que los individuos son políticamente incorporados a la sociedad y a ciertos beneficios modulan no solo las determinantes del BS sino el peso de los elementos del capital social.

René Millán - Doctor en Sociología por la Universidad de Torino, Italia. Investigador del Instituto de Investigaciones Sociales, Universidad Nacional Autónoma de México. E-mail: $<$ renem@sociales.unam.mx>.

\section{Referencias Bibliográficas}

AHN, T. K. "Finitely repeated $2 \times 2$ social dilemma games: equilibrium analysis and experimental test". In: Workshop in Political Theory and Policy Analysis, 2000, Working paper W00-26, Bloomington: Indiana University.

Alesina, A.; DI Tella, R.; MACCulloch, R. "Inequality and happiness: are Europeans and Americans different?". Journal of Public Economics, Amsterdam, n 88, p. 2.009-2.042, 9-10, August 2004.

AXELROD, R. The evolution of cooperation. New York: Basic Books, 1984.

BARTOLINI, S. Sociability predicts happiness in nations: evidence from macro and micro data. In: BARTolini, S. (ed.). Policies for happiness. Oxford: Oxford University Press, 2012.

BeCChettI, L.; GIACHIN, E.; Pelloni, A. "On the causal impact of relational goods on happiness". Center for Economic and International Studies, Roma, vol. 7, issue 5, $n^{\circ}$ 151, November 2009.

Becchetti, L.; Pelloni, A.; Rossettı, F. "Relational goods, sociability, and happiness". Center for Economic and International Studies, Roma, vol. 6, issue 4, n 117, J uly 2008.

BERger, P. (ed.). Los límites de la cohesión social. Barcelona: Galaxia, 1999.

BREHM, J.; RAHN, W. "I ndividual-level evidence for the causes and consequences of social capital". American J ournal of Political Economy, Michigan, vol. 41, n³ 3, p. 999-1023, July 1997.

Cepal. Cohesión social: inclusión y sentido de pertenencia en América Latina y Caribe. Mayo 2007. Disponible en: <http://hdl.handle.net/11362/2812>. Acceso: 17 mar. 2014.

Commission on the Measurement of Economic Performance and Social Progress, 2009. Disponible en: <www.stiglitz-sen-fitoussi.fr>. Acceso: 6 abr. 2014.

ColEman, J. "Social capital in the creation of human capital". American Journal of Sociology, The University of Chicago Press, n 94, p. 95-120, January 1988.

Foundation of social theory. Cambridge, Massachusetts and London: The Belknap Press of Harvard University Press, 1990.

DIENER, E.; OISHI, S. Money and happiness: income and subjective well-being across nations. In: DIENER, E.; SUH, E. (eds.). Culture and subjective well-being. Cambridge, MA: MIT Press, 2000.

Durkheim, E. De la division du travail social. Paris: Press Universitaires de France, 1960. 
EASTERLIN, R. Does economic growth improve the human lot? Some empirical evidence. In: DAVID, P.; Melvin, W. (eds.). National and households in economic growh. New York: New York Academic, 1974.

ELLISON, C. G. "Religious involvement and subjective well-being". Journal of Health and Social Behavior, Sage, $n^{\circ} 32$, p. 80-99, March 1991.

EuRopean CommitTeE for Social Cohesion. A new strategy for social cohesion. 2004. Disponible en: <http://www.coe.int/t/dg3/socialpolicies/socialcohesiondev/source/RevisedStrategy_en.pdf>. Acceso: 12 mayo 2014.

FlorenZANO, R.; DusSAILlANT, F. Felicidad, salud mental y vida familiar. In: RojAS, M. (ed.). La medición del progreso y del bienestar: propuestas desde América Latina. México: Foro Consultivo Científico y Tecnológico, 2011.

FUKUYAMA, F. Trust: the social virtues and the creation of prosperity. New York: The Free Press, 1995.

GarcíA, J.; Fuentes, N.; Borrego, S. M. Values and happiness in Mexico: the case of the metropolitan city of Monterrey. In: BRUNI, L.; PORTA, P. L. (eds.). Handbook of the Economics of Happiness. United Kingdom: Edward Elgar Publishing Limite, 2006.

GARDNER, J.; OsWALD, A. J. "Do divorcing couples become happier by breaking up?". Journal of the Royal Statistical Society, Great Britain, vol. A, n 169, p. 319-336, March 2006.

GoRdON, S.; MILLÁN, R. Análisis preliminar de la cohesión social: un estudio comparativo. México: Instituto de Investigaciones Sociales, Unam, 2009.

Granovetter, M. "The strength of weak ties". American J ournal of Sociology, University Chicago Press, vol. 78, $\mathrm{n}^{\circ}$ 6, p. 1.360-1.380, May 1973.

GuARDIOLA, J.; GARCíA-MUÑOZ, T. "Subjective well-being and basic needs: evidence from rural Guatemala". In: XVI Encuentro de Economía Pública. Editores Universidad de Sevilla, 5-6, Febrero 2009.

GuI, B. "Éléments pour une definition d'économie communautaire", Notes et Documents de I'Institut International Jacques Maritain, Roma, 19/20, p. 32-42, 1987.

GuVEN, C. "Are happier people better citizens?". SOEPpapers, Berlín, n 199, p. 1-51, June 2009.

HARDIN, R. Conceptions and explanations of trust. In: CooK, K. (ed.). Trust in society. New York: Russell Sage, 2001.

HeLliWeLL, J. "How's life? Combining individual variables to explain subjective well-being". NBUR Working Paper, Cambridge, MA, n².065, p. 1-20, July 2002.

Helliwell, J. F.; Barrington-Leigh, C. P. How much is social capital worth? In: Jetten, J.; Haslam, C.; HASLAM, S. A. (eds.). The social cure. London: Psychology Press, 2011.

HELLIWELL, J .; LAYARD, R.; SACHS, J. World happiness report. 2013. Disponible en: <unsdsn.org/wpcontent/.../02/WorldHappinessReport2013_online.pdf>.

Helliwell, J.; Putnam, R. D. "The social context of well-being". Philosophical Transactions of the Royal Society, EEUU, nº, 359(1449), p. 1.435-1.446, September 2004.

Hoogle, M. "I'm happy, hope you're happy too. Examining the different dynamics of individuals subjective well-being view on society". Journal of Happiness Studies, vol. 13, n 1, p. 17-29, 
Belgium, March 2012.

Kollock, P. "Social dilemmas: the anatomy of cooperation". Annual Reviews Social, L.A. California, $n^{\circ} 24$, p. 183-214, August 1998.

LORA, E. (coord.). Calidad de vida. Más allá de los hechos. Resumen Ejecutivo, Banco

Interamericano de Desarrollo, 2008. Disponible en: <http://www.iadb.org/idbdocs/1776313.pdf>.

Melgar, N.; Rossı, M. Percepción individual de bienestar y desempeño macroeconómico. In: Rojas, M. (ed.). La medición del progreso y del bienestar. Propuestas desde América Latina. México: Foro Consultivo Científico y Tecnológico, 2011.

MıLLÁN, R. Complejidad social y nuevo orden en la sociedad mexicana. México: Miguel Ángel Porrúa - II Sunam, 2008.

El bienestar como el nuevo 'objeto' del progreso. Cinco reflexiones. In: RoJ AS, M. (ed.). La medición del progreso y del bienestar: propuestas desde América Latina. México: Foro Consultivo Científico y Tecnológico, 2011.

MILLÁN, R.; GoRdon, S. "Capital social: una lectura de tres perspectivas clásicas". Revista Mexicana de Sociología, México, n 4, p. 711-747, 2004.

MOYANO, E.; RAMOS, N. "Bienestar subjetivo: midiendo satisfacción vital, felicidad y salud en población chilena de la Región Maule". Universum (en línea), vol. 22, n² 2, p. 177-193, 2007. Disponible en:

$<$ http://www.scielo.cl/scielo.php?script=sci_arttext\&pid=S071823762007000200012\&lng=en\&nrm =iso\&ignore=.html, 2007>. Acceso: 26 feb. 2014.

NORTH, D. Instituciones, cambio institucional y desempeño económico. México: Fondo de Cultura Económica, 1993.

OCDE. Guidelines on measuring subjective well-being. OECD Publishing, 2013. Disponible en: <http://dx.doi.org/10.1787/9789264191655>.

OLSON, M. The logic of collective action: public goods and the theory of groups. Cambridge, Massachusetts: Harvard University Press, 1965

Olson, D.; De Frain, J. Marriage and the family: diversity and strengths. Mayfield: Publishing Company, 2000.

Ostrom, E. Understanding institutional diversity. Princeton: Princeton University Press, 2005.

Ostrom, E.; AHN, T. "Una perspectiva del capital social desde las ciencias sociales: capital social y acción colectiva". Revista Mexicana de Sociología, México, n 1, p. 155-233, Agosto 2003.

OT, J. "Greater happiness for a greater number: some non-contraversal options for governments". Journal of Happiness Studies: An interdisciplinary forum on subjective well-being, Berlín, vol. 11, $\mathrm{n}^{\circ}$ 5, p. 631-647, May 2010.

OTTONE, E. Cohesión social, inclusión y sentido de pertenencia en América Latina y el Caribe, Síntesis. Santiago de Chile, Naciones Unidas - Cepal - Aeci - Segyp, 2007.

Portes, A.; LANDolt, P. "The downside of social capital". The American Prospect, vol. 7/26, $n^{\circ} 94$, p. 18-21, May-J une 1996.

PUTNAM, R. Bowling alone: the collapse and revival of American community. Ney York: Simon \& Schuster, 2000. 
RENÉ MILLÁN

Putnam, R.; Goss, K. "Introducción". In: Putnam, R. (ed.). Democracies in flux. The evolution of social capital in contemporary society. Oxford: Oxford University Press, 2002.

Putnam, R., LEONARDI, R., NANETTI, R. Para que la democracia funcione: tradiciones cívicas en Italia. Caracas: Galas, 1994.

RojAS, M.; MARTínEZ, I. (eds.). Medición, investigación e incorporación a la política pública del bienestar subjetivo: América Latina. México: Foro Consultivo Científico y Tecnológico, Octubre 2012.

RoJAS, M., VITTERSO, J. "Conceptual referent for happiness: Cross-country comparisons". Journal of Social Research \& Policy, University of Oradea, n², p. 1-14, September 2010.

Rossı, M.; CID, A.; Ferres, D. Subjective well-being in the Southern Cone: health, income and family. Documentos de trabajo, Departamento de Economía (Econ), Universidad de La República, Uruguay, Mayo 2008.

STOLLE, D. Clubs and congregations: the benefits of joining an association. In: CooK, K. (ed.). Trust in society. New York: Russell Sage, 2011.

ThOITS, P; HeWITT, L. ; Volunteer, L. "Work and well-being". Journal of Health and Social Behavior, Washington D.C., $n^{\circ} 42$, p. 115-131, J une 2001.

TORSVIK, G. "Social capital and economic development". Rationality and Society, Cornell University, vol. 12, $n^{\circ} 4$, p. 451-476, November 2000.

UHLANER, C. J. "Relational goods and participation: incorporating sociability into a theory of rational action". Public Choice, Western Carolina University, vol. 62, n 3, p. 253-285, September 1989.

Veenhoven, R. "The four qualities of life. Ordering concepts and measures of the good life". Journal of Happiness Studies, Kluwer Academic Publishers Netherlands, vol. 1, n 1, p. 1-39, March 2000.

WEITZ-SHAPIRO, R.; WINTER, M. "Political participation and quality of life". Inter-American Development Bank (BID), Research Department, Working Paper \#638, Columbia University, July 2008.

Yamamoto, J.; Feijoo, A. R. "Componentes émicos del bienestar. Hacia un modelo alternativo de desarrollo". Revista de Psicología, Lima, vol. XXV, n², p. 197-231, December 2007.

\section{Resumen}

Integración sociopolítica y bienestar subjetivo en México

Este artículo explora, en dos ciudades, la relación entre integración sociopolítica y bienestar subjetivo. Considera cuatro dimensiones de la integración: cohesión social; capital social; relación entre gobierno y ciudadanía; y calidad de vida. El resultado muestra que las formas de integración sociopolíticas afectan o modulan la asociación, internacionalmente asumida, de la felicidad con distintas variables. Eso significa que los contextos de las comunidades importan en la determinación del bienestar subjetivo. Son ellos los que explican la heterogeneidad del comportamiento o variabilidad de la felicidad. No obstante, hay asociaciones que se confirman más allá de los contextos específicos.

Palabras clave: bienestar subjetivo; felicidad; cohesión social; capital social; bienes relacionales 


\section{Abstract}

Sociopolitical integration and subjective well-being in México

This article explores the relationship between sociopolitical integration and subjective well-being in two cities. Four dimensions of integration are considered: social cohesion, social capital, relationship between government and citizenship, and quality of life. The results show the forms of sociopolitical integration influence or modulate the association, internationally accepted, of happiness with different variables. That means that contexts of communities matter in determining subjective well-being. These contexts explain the heterogeneity of behavior or variability regarding happiness. However, there are associations that are confirmed beyond the specific contexts.

Keywords: subjective well-being; happiness; social cohesion; social capital; relational goods

Artigo submetido à publicação em novembro de 2014. Versão final aprovada em fevereiro de 2015. 